\title{
Evaluation de l'état nutritionnel chez l'enfant âgé de 6 à 59 mois atteint de cancer
}

\author{
Assessment of nutritional status in children aged 6 to 59 months with cancer \\ Abdoul Karim Doumbia ${ }^{1}$, Hawa Diall ${ }^{1}$, Oumar Coulibaly¹, Fatoumata dite Nana Diarra ${ }^{1}$, Adama Dembélé1, Pierre Togo ${ }^{1}$, Mohamed Elmouloud \\ Cissé1, Ibrahima Ahmadou ${ }^{1}$, Karamoko Sacko¹, Fatoumata Léonie Diakité1, Djenéba Konaté1, Bourama Kané2, Lala Ndrany Sidibé1, \\ Fatoumata Dicko Traoré1, Mariam Sylla1 ${ }^{1}$, Boubacar Togo' ${ }^{1}$, Abdoul Aziz Diakité1
}

1. Département de Pédiatrie du CHU-Gabriel Touré

2. Service de pédiatrie de l'Hôpital du Mali

*Auteur correspondant : Abdoul Karim Doumbia, Pédiatre oncologue, CHU Gabriel Touré ; email : doumbiav@gmail.com

\begin{abstract}
Résumé
Introduction: La malnutrition, complication fréquente de l'évolution des cancers est à la fois un problème médical et un problème social. L'objectif était de déterminer les aspects épidémiologiques, cliniques et évolutifs des enfants atteints de malnutrition à l'unité d'oncologie pédiatrique. Méthodologie: II s'agissait d'une étude rétro-prospective sur des patients âgés de moins de 5 ans atteints de malnutrition et suivis dans l'unité d'oncologie pédiatrique (UOP) du 1er janvier au 30 juin 2019. Les données anthropométriques (rapport poids/taille, périmètre brachial) ont été évaluées au moment du diagnostic. La malnutrition était définie par une émaciation accompagnée ou non d'œdème. Résultats : La prévalence de la malnutrition en oncologie pédiatrique était de 34\%. L'âge moyen était de 30 mois avec un sex-ratio de 1. La majorité (94\%) des patients provenaient de milieu socio-économique défavorisé. Le délai moyen de consultation était de 10 semaines. Sur la base de l'indice poids/taille, $63 \%$ des patients avaient une malnutrition sévère et $37 \%$ une malnutrition modérée. Le périmètre brachial était inférieur à la normale dans $81 \%$ des cas. Les cancers les plus fréquents étaient le rétinoblastome, la leucémie et le néphroblastome. Le taux de mortalité était de $44 \%$. Conclusion: Les enfants atteints de cancer sont à haut risque de dénutrition, d'où l'importance de déterminer les différents facteurs de risque et d'élaborer un protocole de prise en charge nutritionnelle dans leurs protocoles thérapeutiques.
\end{abstract}

Mots clés : Cancer, nutrition, évaluation, pédiatrie.

\begin{abstract}
Introduction: Malnutrition, a frequent complication of cancer progression, is both a medical problem and a social problem. The objective was to determine the epidemiological, clinical and evolutionary aspects of children with malnutrition at the pediatric oncology unit. Methodology: This was a retro-prospective study on patients aged less than 5 years suffering from malnutrition and followed in the POU from January 1 to June 30, 2019. Anthropometric data (weight / height ratio, MUAC ) were assessed at the time of diagnosis. Malnutrition was defined
\end{abstract}

by emaciation with or without edema. Results: The prevalence of malnutrition in pediatric oncology was $34 \%$. The mean age was 30 months with a sex ratio of 1 . The majority (94\%) of the patients were from low socioeconomic backgrounds. The average consultation time was 10 weeks. Based on the weight for height index, $63 \%$ of patients had severe malnutrition and $37 \%$ moderate malnutrition. MUAC was below normal in $81 \%$ of cases. The most common cancers were retinoblastoma, leukemia and nephroblastoma. The death rate was $44 \%$. Conclusion: Children with cancer are at high risk of malnutrition, hence the importance of determining the different risk factors and developing a nutritional management protocol in their treatment protocols.

Keywords: Cancer, nutrition, evaluation, pediatrics

\section{A. INTRODUCTION}

La malnutrition constitue un problème majeur chez l'enfant atteint de cancer [1]. Le nombre de nouveaux cas de cancer est en constante évolution en Afrique et plus de $95 \%$ des cas sont diagnostiqués à un stade avancé [2]. Au Mali, il n'existe pas de registre pour les cancers pédiatriques. Selon une étude antérieure, la majorité des cancers pédiatriques survient avant l'âge de 5 ans [3] ;les cancers fréquemment diagnostiqués étaient les lymphomes non hodgkiniens $(33,5 \%)$, le rétinoblastome $(24,6 \%)$, le néphroblastome $(14,8 \%)$, les leucémies aiguës lymphoblastiques (7\%) et la maladie de Hodgkin (4\%) [3]. La malnutrition était le plus souvent observée au moment du diagnostic du cancer [3]. Selon la littérature, la prévalence de la dénutrition en oncologie pédiatrique est estimée entre 10 et $46 \%$ chez les enfants atteints de tumeurs métastatiques $[1,4,5]$. L'état nutritionnel des enfants atteints de cancer est donc une préoccupation générale. II dépend du type de cancer, de leur localisation, du stade, de l'intensité de la chimiothérapie utilisée et de la définition utilisée $[1,6]$. Une nutrition adéquate joue un rôle déterminant dans la réponse à la chimiothérapie, la qualité de vie et diminue le coût des soins anticancéreux [5, 6]. Dans des pays en développement, où vivent $85 \%$ des enfants du monde, l'état de santé est fréquemment influencé négativement par les maladies infectieuses et la malnutrition $[7,8]$. L'impact de la malnutrition en oncologie pédiatrique est sous-évalué. La présente étude a été initiée dans le but d'évaluer la prévalence de la dénutrition, d'identifier les 
cancers à haut risque de malnutrition et de dépister les enfants à risque élevé de dénutrition.Les données obtenues permettront de renforcer la collaboration, l'échange de connaissances et de compétences entre oncologues pédiatriques et nutritionnistes.

\section{B. METHODOLOGIE}

Notre étude s'est déroulée à l'unité d'oncologie pédiatrique et à l'unité de Récupération et d'Education Nutritionnelle Intensive (URENI) du CHU Gabriel Touré. L'UOP est le seul site de prise en charge des enfants atteints de cancer dans notre pays. II comporte dix (10) salles d'hospitalisation individuelle, une salle pour la préparation de la chimiothérapie, une infirmerie, une salle de consultation. Le personnel de l'UOP est constitué de cinq infirmières, quatre oncologues-pédiatres et un médecin généraliste. Le personnel de l'URENI est constitué d'un pédiatre superviseur, et de trois techniciennes de santé.

II s'agissait d'une étude retro-prospective qui s'est déroulée du 1er janvier 2019 au 30 juin 2019 soit une durée de 6 mois. Notre étude a concerné les enfants âgés de 0 à 59 mois atteints de cancer et suivis à l'unité d'oncologie pédiatrique. Les critères d'inclusion étaient :

- Enfants suivis en oncologie pédiatrique, au $\mathrm{CHU}$ Gabriel Touré,

- Présentant un cancer,

- $\quad$ Pris en charge du 1 er janvier 2019 au 30 juin 2019,

- $\quad$ Age compris entre 0 à 59 mois.

La collecte des données était faite à l'aide d'un questionnaire préétabli. Les dossiers médicaux avaient été revus rétrospectivement pour recueillir les données constatées à l'admission : âge, sexe, profession et niveau d'instruction des parents, la résidence, les circonstances de découverte du cancer, le type de cancer. Les informations sur l'état nutritionnel (rapport poids/taille, rapport taille/âge, rapport poids/âge ; périmètre brachial) et le devenir des patients avaient été recueillies à l'interrogatoire de manière prospective.

Le statut nutritionnel a été mesuré au moment du diagnostic. Pour classer l'état nutritionnel clinique, nous avons utilisé les courbes poids pour taille, taille pour âge, poids pour âge Z scores recommandées par l'OMS (2007) et le périmètre brachial pour les $\leq 5$ ans. La malnutrition était définie par rapport au poids/taille [ $\geq-3$ à $<-2$ z-score (malnutrition modérée), $<-3$ z-score (malnutrition sévère)] ou au périmètre brachial : < 115 (malnutrition sévère) ; $115 \leq \mathrm{PB}$ $<125$ (malnutrition modérée)[8]. Chez les enfants atteints de tumeurs solides ou d'œdème, le poids peut être trompeur. Le périmètre brachial est alors recommandé pour l'évaluation de la malnutrition [8]. Le diagnostic de cancer était retenu après une confirmation anatomopathologique. Les données ont été saisies sur Microsoft world 2016 et ont été analysées sur le logiciel SPSS 20.

\section{RESULTATS}

Sur 47 patients de moins de cinq ans suivis à I'UOP, 16 souffraient de malnutrition, soit une prévalence de $34 \%$. Les principales caractéristiques sociodémographiques et les signes révélateurs du cancer et de la malnutrition sont rapportés dans le Tableau I. Les enfants de moins de 36 mois représentaient $62 \%$ et les nourrissons (moins de 24 mois) représentaient $31 \%$ des patients. Le sex-ratio était de 1. Quarante-quatre pour cent vivaient dans la zone urbaine de Bamako et $80 \%$ provenaient d'un milieu socioéconomique défavorable. Les mères étaient le plus souvent des ménagères (94\%) et non scolarisées dans $75 \%$ des cas. Le délai moyen de consultation était de 10 semaines (extrêmes : 4 et 48 semaines). La plupart des enfants avait reçu un allaitement maternel exclusif jusqu'à 6 mois (94\%). La diversification alimentaire avait débuté avant l'âge de 7 mois chez $63 \%$ des patients. Les patients recevaient au moins 4 repas journaliers (56\%). Les principaux signes cliniques étaient les plis de dénutrition (38\%), la masse abdominale (31\%) et l'enfoncement des yeux dans 19\% des cas.

Le périmètre brachial était inférieur à la normale $(<125 \mathrm{~mm})$ dans $81 \%$ des cas. Le périmètre brachial était $<115 \mathrm{~mm}$ chez $75 \%$ des patients atteints de néphroblastome. Le Zscore était inférieur à -3 dans $38 \%$ des cas. Dans notre étude, $63 \%$ des patients avaient une malnutrition aiguë sévère et $37 \%$ une malnutrition modérée. Le rétinoblastome, la leucémie et le néphroblastome (75\%) étaient les principaux types de cancers rapportés chez les patients atteints de malnutrition (Tableau II).

Les patients avaient été soumis à une chimiothérapie intensive dans $87 \%$ des cas.lls étaient suivis à l'unité de récupération et d'éducation nutritionnelle intensive (URENI) dans $75 \%$ des cas et dans l'unité de récupération et d'éducation nutritionnelle ambulatoire sévère (URENAS) dans $25 \%$. Le Plumpy-nut était prescrit dans $56 \%$ des cas et le lait thérapeutique (F75 ou F100) dans 19\%. Une chirurgie invasive avait été réalisée dans $56 \%$ des cas, une transfusion dans $56 \%$ et une antibiothérapie dans $38 \%$. La durée du séjour à l'URENI était supérieure à 2 semaines dans $73 \%$ des cas. Dans notre étude, $44 \%$ des enfants étaient décédés.

Dans le tableau III, l'évolution des patients est expliquée en détail. L'abandon de traitement était constaté chez les enfants de pères paysan ou ouvrier.

\section{DISCUSSION}

Les limites de l'étude étaient liées à son caractère rétroprospectif, monocentrique et à l'insuffisance de données biologiques pour la définition des cas. Dans notre étude, la prévalence de la malnutrition à l'admission (34\%) était comparable à celle retrouvée dans la littérature (10 à $45 \%$ ) [1]. Des taux plus importants ont été signalés en Australie 
par Murphy et al (45\%) dans une étude réalisée en 2010 [9]. L'estimation de la prévalence de la malnutrition dépend des marqueurs choisis pour la définir, et de la population étudiée (type de tumeur, stade évolutif, traitements mis en œuvre, etc.) [10].

Dans notre étude, l'évaluation nutritionnelle des patients était réalisée dès leur admission. Habituellement, l'état nutritionnel est défini selon les critères de Waterlow, ou en calculant l'indice de masse corporelle (IMC en $\mathrm{kg} / \mathrm{m}^{2}$ ) qui est un bon reflet de la composition du corps [6, 8]. Le principal avantage de cette méthode est sa simplicité mais elle peut être source d'erreur notamment chez les nourrissons avec de grosses tumeurs. II est alors préférable d'utiliser d'autres mesures anthropométriques comme le périmètre du bras ou l'épaisseur du pli cutané tricipital [6]. En cancérologie pédiatrique, l'utilisation du poids comme indicateur de dénutrition peut entrainer des erreurs de classement car les masses tumorales peuvent parfois atteindre $10 \%$ du poids du corps et il peut exister un œdème [1]. Le périmètre brachial est donc recommandé dans ces situations et sa prise régulière pourrait aider à prévenir un retard de croissance chez l'enfant atteint de tumeur solide [8, 12]. Les critères biologiques pour l'évaluation nutritionnelle notamment le dosage du taux de préalbumine sont discutés $[1,13]$, mais il n'y a pas de consensus et leur pratique au quotidien n'est pas courante selon Martin E. et al. L'évaluation de la croissance est donc l'outil le plus utile pour définir l'état de santé et l'état nutritionnel d'un enfant atteint de cancer [1,13]. Elle doit donc être réalisée le plus tôt possible et répétée régulièrement afin d'adapter la prise en charge nutritionnelle.

Au Mali, le groupe à risque de malnutrition et de cancer est celui des enfants de moins de 5 ans $[3,8,14]$. Plusieurs auteurs estiment que la prévalence de la malnutrition pédiatrique pourrait dépasser $50 \%$ dans les pays aux ressources limitées [9]. Dans notre étude, le sex-ratio était de 1 alors que la littérature précise que le sexe masculin serait plus associé à un risque accru de cancer pédiatrique[3, 15]. La majorité de nos patients provenait de milieu socio-économique défavorisé. L'éducation, la profession des parents et le revenu familial sont généralement des facteurs de survenue de la dénutrition dans une communauté $[16,17]$. La plupart de nos enfants étaient sous allaitement maternel exclusif jusqu'à l'âge de 6 mois (94\%). Selon des chercheurs suédois, il n'existe aucune association entre la durée de l'allaitement et les cancers pédiatriques; mais des études plus récentes précisent que l'allaitement avait un effet protecteur contre certains types de cancer pédiatrique (neuroblastome, leucémie) $[18,19]$. Des auteurs indiens affirment même que le taux élevé d'allaitement au sein pourrait expliquer la faible incidence du cancer chez les enfants indiens [20].

Dans notre étude, les principaux signes révélateurs de la maladie cancéreuse étaient la leucocorie et la masse abdominale (31\% et $25 \%$ ). Des signes peu spécifiques (adénopathies, douleur abdominale et pâleur) étaient aussi observés. Une étude britannique avait révélé que près de $60 \%$ des patients atteints de cancer étaient monosymptomatique [21].

La malnutrition et le cancer rendent les enfants plus vulnérables face aux infections tropicales. Les cancers et leur traitement sont sources de complications digestives (nausées, vomissements, diarrhée, occlusion) ou extradigestives (douleurs, anorexie, asthénie) favorisant la dénutrition. Les principaux types de cancers retrouvés dans notre étude étaient le rétinoblastome, la leucémie et le néphroblastome. Nos résultats étaient différents de ceux obtenus par la plupart des auteurs d'Afrique Subsaharienne compte tenu de la différence de méthodologie [22]. Selon la littérature, la proportion de patients souffrant d'insuffisance pondérale varie considérablement en fonction du type de cancer [23]. En oncologie, les enfants à haut risque de malnutrition sont ceux atteints de cancers métastatiques ou de cancers traités par une chimiothérapie intensive [6]. Dans l'étude de Murphy et al, les enfants atteints de tumeurs hématologiques avaient plutôt tendance à l'obésité due aux corticostéroïdes utilisés dans les protocoles de traitement [9]. Ceux atteints de tumeurs solides deviennent malnutris à cause de la charge tumorale et du traitement médicamenteux [9].

Dans notre étude, la prise en charge nutritionnelle avait consisté essentiellement à la supplémentation par les aliments thérapeutiques (lait thérapeutique et Plumpy-nut). Le soutien nutritionnel est indispensable pour prévenir ou traiter la malnutrition et améliorer le bien-être des enfants atteints de cancer. Les interventions nutritionnelles ciblées pour les groupes à haut risque pourraient améliorer la morbidité et la mortalité comme cela a été démontré au Malawi, où un taux élevé de malnutrition aiguë a été observé chez les patients atteints de néphroblastome au moment du diagnostic (45-55\%) [23, 24]. L'étiologie de la malnutrition chez les enfants atteints de cancer est multifactorielle [12]. Le cancer et son traitement sont des facteurs contribuant à la diminution de l'apport digestif et au développement de la malnutrition [5, 12, 25]. La réussite de la prise en charge passe par la reconnaissance et la résolution des problèmes médicaux et sociaux. L'objectif du traitement étant la guérison, le patient devrait bénéficier d'une prise en charge nutritionnelle et d'un traitement anticancéreux optimal. La récupération nutritionnelle permettra alors de réduire les complications du cancer et du traitement et d'améliorer la qualité de vie du patient [22]. Les taux de décès et d'abandon de traitement étaient considérablement élevés dans notre étude (44\% et $25 \%$ ). Ceux-ci seraient surtout liés en l'absence de soutien psychologique et économique aux familles déjà affectées par la pauvreté. La dénutrition apparait clairement comme un élément pronostic majeur de l'évolution des cancers dans notre contexte [8] où 
il n'existe pas de politique nationale de lutte contre le cancer pédiatrique [26].

\section{E. CONCLUSION}

Le cancer et la malnutrition constituent un problème de santé publique avec une prévalence estimée à $34 \%$. La majorité des patients avait moins de 36 mois avec un sexratio de 1. Ils étaient le plus souvent issus de milieu socioéconomique défavorisé. Les cas de rétinoblastome étaient les plus fréquents. L'évaluation nutritionnelle doit être systématique chez tous les enfants atteints de cancer. Leur prise en charge doit comporter nécessairement une intervention nutritionnelle pour améliorer leur pronostic. II est donc nécessaire d'organiser des campagnes de sensibilisation sur les cancers de l'enfant plus précisément dans les zones rurales.

Les conflits d'intérêts

Les auteurs ne déclarent aucun conflit d'intérêts concernant la publication de cet article.

\section{F. REFERENCES}

1. Martin E, Belleton F, Lallemand $Y$, Goy $F$ et al. Dénutrition en cancérologie pédiatrique : prévalence et dépistage. Archives de pédiatrie. 2006;13(4):352-357.

2. Brahmi SA, Ziani FZ, Seddik Y, Afqir S. [Medical oncology: is it a new medical speciality in Africa?] The Pan African Medical Journal. 2017 ;27:36. DOl: 10.11604/pamj.2017.27.36.9531.

3. Togo B, Traore F, Togo A. P, Togo P, Diakitè A.A, Traore $\mathbf{B}$ et al. Epidémiologie et pronostique des cancers pédiatrique au CHU Gabriel Touré de Bamako Mali.Médecine et Santé Tropicales. $2014 ; 24$ :68-72.

4. Barr R D. Nutritional status in children with cancer: Before, during and after therapy. Indian $\mathrm{J}$ Cancer. 2015;52:173-175.

5. Bauer J, Jürgens $\mathbf{H}$, Frühwald MC. Important aspects of nutrition in children with cancer. AdvNutr. 2011;2(2):67-77.

6. Minard-Colin Véronique, Jacques Grill. Stratégies de prise en charge nutritionnelle spécifiques : l'enfant en cancérologie. Nutrition clinique et métabolisme. 2005;19(4):234-237.

7. Sala A, Pencharz P, Barr RD. Children, cancer, and nutrition - A dynamic triangle in review. Cancer. 2004;100:677-87.

8. Schoeman J. Nutritional assessment and intervention in a pediatric oncology unit. Indian $\mathrm{J}$ Cancer 2015;52:186-90

9. Murphy AJ, White M, Davies PS. Body composition of children with cancer. Am J Clin Nutr 2010; 92:55-60.
10. Colomb V. Nutrition en cancérologie : aspects pédiatriques. Nutrition Clinique et Métabolisme. $2001: 15(4): 325-334$.

11. Ministère de la Santé et de l'Hygiène Publique de la République du Mali / Direction Nationale de la Santé(2017) Protocole de Prise en Charge Intégrée de la Malnutrition Aigue au Mali. 204 p.

12. Antoun S, Baracos VE. Comment dépister la dénutrition au cours du cancer ? Oncologie, $2009 ; 11(4): 211-217$.

13. Elhasid R, Laor A, Lischinsky $\mathbf{S}$, et al. Nutritional status of children with solid tumors. Cancer. 1999;86:119-25.

14. Institut National de la Statistique (INSTAT), Cellule de Planification et de Statistique Secteur SantéDéveloppement Social et Promotion de la Famille (CPS/SS-DS-PF) et ICF. 2019. Enquête Démographique et de Santé au Mali 2018. Bamako, Mali et Rockville, Maryland, USA : INSTAT, CPS/SSDS-PF et ICF.

15. Williams LA, Richardson M, Kehm RD, et al. The association between sex and most childhood cancers is not mediated by birthweight. Cancer Epidemiol. 2018;57:7-12.

16. Duru CB, Oluoha UR, Uwakwe KA, Diwe KC, Merenu IA, Chigozie IO, Iwu AC. Prévalence et déterminants sociodémographiques de la malnutrition chez les enfants de moins de cinq ans dans les communautés rurales de l'État d'Imo. Nigeria Am J Pub Health Res. $2015 ; 3: 199-206$.

17. Galgamuwa LS, Iddawela D, Dharmaratne SD, Galgamuwa GLS. Nutritional status and correlated socio-economic factors among preschool and school children in plantation communities, Sri Lanka. BMC Public Health. 2017;17(1):377.

18. Hardell $L$ and Dreifaldt $A C$. Breastfeeding duration and the risk of malignant diseases in childhood in Sweden. Eur J Clin Nutr. 2001;55:179-185.

19. Gao Z, Wang R, Qin ZX, Dong A, Liu CB. Protective effect of breastfeeding against childhood leukemia in Zhejiang Province, P. R. China: a retrospective casecontrol study. Libyan J Med. 2018; 13(1):1508273.

20. Mathur GP, Gupta N, Mathur S, Gupta V, Pradhan S, Dwivedi JN, et al. Breastfeeding and childhood cancer. Indian Pediatr. 1993 May; 30(5):651-7.

21. Ahrensberg JM, Hansen RP, Olesen F, Schrøder H, Vedsted P. Presenting sssymptoms of children with cancer: a primary-care population-based study. $\mathrm{Br} \mathrm{J}$ Gen Pract. 2012; 62(600):e458-e465.

22. Ongotsoyi AHP, Kamdem AKB, Kamgaing N, Mbog GJN, Adiogo D. Evaluation de l'État Nutritionnel chez les Enfants Atteints de Cancers Hospitalisés au Centre 
Mère et Enfant de Yaoundé. HEALTH SCIENCES AND DISEASE. $2018 ; 19(4): 42-46$.

23. ISRAËLS T, BORGSTEIN E, JAMALI M, DE KRAKER J, CARON HN, MOLYNEUX EM. ACUTE MALNUTRITION IS COMMON IN MALAWIAN PATIENTS WITH A WILMS TUMOUR: A ROLE FOR PEANUT BUTTER. PEDIATR BLOOD CANCER. $2009 ; 53(7): 1221-1226$.

24. Elena J Ladas, Marc Gunter, Inge Huybrechts et Ronald Barr , A Global Strategy for Building Clinical Capacity and Advancing Research in the Context of Malnutrition and Cancer in Children in Low- and MiddleIncome Countries , JNCl Monographs , 10.1093 / jncimonographs / lgz023 , 2019 , 54 , (149151) , (2019).

25. WHO Antro 2005. Software for Assessing Growth and Development of the World's Children. Geneva: WHO; 2011. Available from: http://www.who.int/childgrowth/software/en. [Last accessed on 2016 Jan 02], [Lats updated on 2011 Jan], [Lats cited on 2014 Aug 22]

26. Goumbri OM., Domagni OE., Sanou AM., Konsegre V., Soudre RB. (2009). Aspects épidémiologiques et histopathologiques des cancers au Burkina Faso. Journal africain du cancer/African Journal of Cancer. $2009 ; 1(4): 207$.

Liste des tableaux

Tableau I. Caractéristiques sociodémographiques et cliniques des enfants.

\begin{tabular}{|c|c|c|c|}
\hline Variables & & Effectif & $\%$ \\
\hline \multirow{2}{*}{ Sexe } & Féminin & 8 & 50 \\
\hline & Masculin & 8 & 50 \\
\hline \multirow{3}{*}{ Age } & 6 à 23 mois & 5 & 31 \\
\hline & 24 à 35 mois & 5 & 31 \\
\hline & 36 à 59 mois & 6 & 38 \\
\hline \multirow{8}{*}{$\begin{array}{l}\text { Symptômes } \\
\text { révélateurs }\end{array}$} & Leucocorie & 5 & 31 \\
\hline & Masse abdominale & 4 & 25 \\
\hline & Adénopathies & 2 & 13 \\
\hline & Céphalées & 1 & 6 \\
\hline & Douleur abdominale & 1 & 6 \\
\hline & Fièvre & 1 & 6 \\
\hline & Strabisme & 1 & 6 \\
\hline & Vomissement & 1 & 6 \\
\hline \multirow{3}{*}{$\begin{array}{l}\text { Périmètre } \\
\text { brachial }\end{array}$} & $<115 \mathrm{~mm}$ & 6 & 38 \\
\hline & $\geq 115 \mathrm{à}<125 \mathrm{~mm}$ & 7 & 44 \\
\hline & $\geq 125 \mathrm{~mm}$ & 7 & 19 \\
\hline \multirow{4}{*}{$\begin{array}{l}\text { Rapport } \\
\text { poids /taille }\end{array}$} & $\begin{array}{l}\text { entre -3 et }-2 \text { z- } \\
\text { scores }\end{array}$ & 6 & 38 \\
\hline & $<-3$ Z score & 6 & 38 \\
\hline & Z score normal & 4 & 24 \\
\hline & Malnutrition modéré & 6 & 38 \\
\hline
\end{tabular}

\begin{tabular}{lccc}
\hline Variables & Effectif & $\%$ \\
\hline $\begin{array}{l}\text { Type de } \\
\text { malnutrition }\end{array}$ & Malnutrition sévère & 10 & 63 \\
\hline
\end{tabular}

Tableau II : Répartition des patients selon le type de cancer

\begin{tabular}{lcc}
\hline Variables & Effectif & $\%$ \\
\hline Rétinoblastome & 6 & 38 \\
Leucémie & 3 & 19 \\
Néphroblastome & 3 & 19 \\
Médulloblastome & 2 & 13 \\
Lymphome de Burkitt & 1 & 6 \\
Hépatoblastome & 1 & 6 \\
Total & 16 & 100 \\
\hline
\end{tabular}

Tableau III : Répartition des patients selon l'évolution

\begin{tabular}{lcc}
\hline Evolution & Effectif & $\%$ \\
\hline Absence de rémission & 10 & 63 \\
Rémission complète & 6 & 38 \\
& & \\
Abandon de traitement & 3 & 19 \\
Perdu de vue & 1 & 6 \\
Décès & 7 & 44 \\
\hline
\end{tabular}

